\title{
RatFish: A File Sharing Protocol Provably Secure against Rational Users
}

\author{
Michael Backes ${ }^{1,2}$, Oana Ciobotaru ${ }^{1}$, and Anton Krohmer ${ }^{1}$ \\ 1 Saarland University \\ 2 MPI-SWS
}

\begin{abstract}
The proliferation of $\mathrm{P} 2 \mathrm{P}$ computing has recently been propelled by popular applications, most notably file sharing protocols such as BitTorrent. These protocols provide remarkable efficiency and scalability, as well as adaptivity to dynamic situations. However, none of them is secure against attacks from rational users, i.e., users that misuse the protocol if doing so increases their benefits (e.g., reduces download time or amount of upload capacity).

We propose a rigorous model of rational file sharing for both seeders and leechers, building upon the concept of rational cryptography. We design a novel file sharing protocol called RatFish, and we formally prove that no rational party has an incentive to deviate from RatFish; i.e., RatFish constitutes a Nash equilibrium. Compared to existing file sharing protocols such as BitTorrent, the tracker in RatFish is assigned additional tasks while the communication overhead of a RatFish client is kept to a minimum. We demonstrate by means of a prototype implementation that RatFish is practical and efficient.
\end{abstract}

\section{Introduction}

Recently, the peer-to-peer (P2P) paradigm has emerged as a decentralized way to share data and services among a network of loosely connected nodes. Characteristics such as failure resilience, scalability and adaptivity to dynamic situations have popularized $\mathrm{P} 2 \mathrm{P}$ networks in both academia and industry. The proliferation of $\mathrm{P} 2 \mathrm{P}$ computing has also been propelled by popular applications, most notably file sharing protocols such as BitTorrent [5].

A crucial assumption underlying the design of such file sharing protocols is that users follow the protocol as specified; i.e., they do not try to bypass the design choices in order to achieve higher download rates, or to avoid uploading to the system at all. However, not all users are necessarily altruistic, and publicly available, modified BitTorrent clients like BitThief [19] or BitTyrant 21] can be used to strategically exploit BitTorrent's design to achieve a higher download while contributing less or nothing at all in return. While several minor protocol adaptations have been suggested to mitigate the attacks underlying these clients [28, the core weaknesses remain: In its current form, BitTorrent - and current file sharing protocols in general - offer better service to cheating clients, thereby creating incentives for users to deviate from the protocol; in turn, it

D. Gritzalis, B. Preneel, and M. Theoharidou (Eds.): ESORICS 2010, LNCS 6345, pp. 607 625, 2010.
(C) Springer-Verlag Berlin Heidelberg 2010 
further decreases the performance of honest clients. The task is thus to design a protocol that not only retains the remarkable characteristics of current file sharing protocols, but that is rational in the sense that it offers sufficient incentives for users to stick to the precise protocol specification. In more technical terms, this file sharing protocol should constitute an equilibrium state: Adhering to the protocol should optimize the benefits received by each individual participant, and any deviation from the protocol should result in a lower payoff for the cheating user.

\subsection{Our Contribution}

We contribute RatFish, a protocol for rational file sharing. RatFish is built upon the concepts and design choices that underlie BitTorrent, but it resolves the weaknesses that BitThief and BitTyrant exploit. We achieve this mostly by ensuring fair exchange of pieces between leechers and by having the tracker participate in the coordination of the downloads.

The distinctive feature of RatFish, however, is not that it discourages the use of several selfish strategies, but that it comes with a formal proof that deviating from RatFish is irrational for both seeders and leechers. In order to do this, we first characterize rational behaviors of leechers and seeders in file sharing protocols, building upon the concept of the recently emerging field of rational cryptography, in which users are defined as rational players in a game-theoretic sense. Intuitively, leechers are primarily interested in minimizing their download time and the amount of uploaded data, whereas seeders value the efficiency of the protocol in using their upload capacity. We cast this intuition into a rigorous mathematical model, and we formally prove that our protocol is secure against deviations of rational parties, by showing that it constitutes a Nash equilibrium. This holds even though RatFish allows dynamically leaving and (re-)joining users. We prove this Nash equilibrium using a new proof technique that is of independent interest for rational cryptography: the step-by-step substitution of a deviating strategy with hybrid, semi-rational strategies.

We have built a prototype implementation of RatFish that demonstrates that RatFish is practical and efficient. We stress though that the purpose of RatFish is not to achieve performance improvements over existing protocols, but to establish a formal proof that under realistic conditions, such as dynamically joining users, no rationally-behaving user has an incentive to deviate from RatFish. The additional computational overhead of RatFish compared to BitTorrent is small: basic cryptographic primitives (symmetric encryptions and digital signatures schemes) are used, and the tracker is assigned additional tasks such as the coordination of downloads and the generation of user incentives. The communication overhead of a RatFish client is kept to a minimum.

\subsection{Related Work}

The performance of BitTorrent has been thoroughly studied 243112322]. All these works attest to the impressive performance of BitTorrent in the presence 
of honest participants; however, 3. 3. has noted that the rate-based Tit-For-Tat policy of BitTorrent does not prevent nodes from uploading less content than they should serve (in all fairness), thereby creating an incentive for abuse of the protocol.

The behavior of BitTorrent in the presence of cheating peers was subsequently investigated [18211926], revealing that cheating leads to a loss in overall performance for honest peers.

Our rigorous model of rational file sharing is grounded in the recently emerging field of rational cryptography, where users are assumed to only deviate from a protocol if doing so offers them an advantage. Rational cryptography is centered around (adapted) notions of game theory such as computational equilibria [6] . A comprehensive line of work already exists that develops novel protocols for important cryptographic primitives such as rational secret sharing and rational secure multiparty computation [7/091148].

In this spirit, there has been a variety of research aimed at making BitTorrent more robust against deviations of rationally-behaving users [28 15 22 20 27]. All these works provide stronger user incentives: they choke problematic connections [28, grant additional bandwidth to generously uploading neighbors [15], reward leechers that continue seeding after their download is completed [22, optimally distribute a seeder's bandwidth across swarms [20], and employ fair exchange protocols to stop leechers from aborting the protocol 27] early. These modified protocols, however, are still prone to rational attacks; in particular, none of these works reached a (Nash) equilibrium 1

The first work that strived to establish an equilibrium in the context of file sharing is 24]. However, this equilibrium was severely restricted in that it was only guaranteed when rational parties were allowed to only tweak the protocol parameters, but not when they could deviate in larger ways.

More recent research such as BAR-B [2], Equicast [13], and FOX [25] aimed at deploying incentives and punishments such that obeying the protocol is the best strategy for every rational player. The first two protocols were shown to be strict Nash equilibria, i.e., a rational peer obtains no benefit from unilaterally deviating from the assigned strategy. The drawback is that their strict equilibrium solutions limit the design: the BAR-B system only permits a static set of users. Equicast requires the rate at which leechers join to precisely match the rate of which they leave and considers only restricted utility functions that do not take downloading time into account; moreover, these protocols require nodes to waste network bandwidth by sending garbage data to balance bandwidth consumption. 25 establishes a stable Nash equilibrium, but again it only allows a static set of leechers; moreover, its rationality is not grounded on incentives but on fear of retaliation such that a single Byzantine node can cause the entire system to collapse. Somewhat orthogonal to our work are the file streaming applications

1 [27] contains proofs that certain deviations from selfish leechers or attacks of malicious peers cannot succeed (e.g., no peer can assume another authorized peer's identity), but there is no equilibirum proof, i.e., that deviating from the protocol cannot yield better results. 
BAR-Gossip [17] and FlightPath [16]. Both works show a Nash equilibrium (a strict one for BAR-GOSSIP, and an approximate one for Flightpath), but rational players are only interested in minimizing the amount of uploaded data and reducing jitter. While such time-independent utility functions are reasonable for streaming applications, they do not apply to the more sophisticated setting of rational file sharing, where minimizing the time to complete a download is usually the primary goal. Moreover, none of these five protocols considers seeders as part of the rational model. We conclude by saying that like our approach, none of these works offers resistance against Sybil attacks 2

\subsection{Outline}

Section 2 provides a bird's eye view of the core ideas underlying how we create incentives in file sharing. Section 3 summarizes the concepts we use from rational cryptography and defines rational behaviors of seeders and leechers. Section 4 presents the RatFish protocol in detail. Section 5 contains the proof of equilibrium for RatFish; i.e., it shows that users cannot achieve a better payoff by deviating from the protocol. Section 6 discusses our experimental results. Section 7 concludes.

\section{A Bird's Eye View on How to Rationalize P2P}

For the sake of exposition, we provide a high-level overview of the core ideas underlying how we create incentives in file sharing. We briefly discuss which behaviors of seeders and leechers we consider rational, intuitively explain how to incentivize these behaviors, and finally discuss how an equilibrium is obtained for a small example protocol. In this section, we abstract away many important systems details and impose several assumptions to improve understanding.

In the following, we consider a single seeder $S$ that intends to upload a file $f$ to leechers $L_{1}, \ldots, L_{M}$. The file is split into pieces $f_{1}, \ldots, f_{M^{2}}$. In this exposition, we describe a simplistic protocol that proceeds in a sequence of $M+1$ monolithic rounds. We assume that the seeder can upload exactly $M$ pieces per round and that every leecher is able to upload and to download at least $M$ pieces of the file in each round.

On the Rationality of Seeding. A seeder is a player that uploads without requesting reciprocation. Intuitively, it thus acts rationally if it uses its upload time and upload speed as efficiently as possible; i.e., for any fixed upload speed and time that the seeder spends within the system, the average download time for all leechers should be as small as possible. It is thus in the interest of the seeder to incentivize leechers to share parts of the file amongst each other as this

\footnotetext{
${ }^{2}$ A Nash equilibrium ensures that no individual user has an incentive to deviate. However, it conceptually does not take coalitions of users into account, rendering Sybil attacks possible in most works on rationally secure protocols.
} 
increases the throughput of the whole system 3 In the simplistic protocol, the seeder sends to each leecher $L_{i}$ in each round $j$ the piece $f_{j \cdot M+i}$.

On the Rationality of Leechers. Leechers aim to download the file as fast as possible while saving upload capacity. The protocol thus has to enforce leecher participation as they will otherwise just download and leave. We need to propose a suitable piece selection algorithm and a piece exchange mechanism that prevents parties from cheating each other. In our example, piece selection is easy: In each round $j$ a leecher $L_{i}$ holds a piece $f_{j \cdot M+i}$ obtained from the seeder that no one else has. As the leecher can upload $M$ pieces per round, he can exchange with the rest of the leechers their unique pieces. To ensure fair exchanges, leechers first exchange the pieces in encrypted form and subsequently send the corresponding decryption keys.

How an Equilibrium is Achieved. We conclude with some basic intuition on why no rational user has an incentive to deviate from the protocol. If all peers adhere to the protocol, the seeder will upload the file exactly once and stay in the system for $M$ rounds. Each of the leechers will upload $M^{2}-M$ pieces and complete its download after $M+1$ rounds. It is easy to see that the average download time and hence the seeder's utility cannot be improved.

This outcome cannot be further improved for the leechers either: None of the leechers can download the file in less than $M+1$ rounds since after round $M$ each of them is missing at least $M-1$ pieces 4 Moreover, since the seeder only provides $M$ pieces to each of its peers, no leecher can obtain the full file without uploading at least $M^{2}-M$ pieces in exchange for the pieces that it is missing from the seeder. This statement holds as no leecher can cheat during the fair piece exchange protocol: A leecher spends his upload capacity to receive an encrypted piece, hence he has no incentive not to send the much smaller decryption key to its partner. Thus, no party can improve its utility by deviating from the protocol.

\section{A Game-Theoretic Model for File Sharing}

In this section, we propose a game-theoretic model for rationally secure file sharing. We start by reviewing central concepts from game theory and rational cryptography.

${ }^{3}$ As a consequence, the naive approach of uploading the whole file to an arbitrary leecher at once cannot yield a rationally secure protocol: This leecher may just complete the download and leave, causing some pieces of the file to be effectively lost from the system. Moreover, since there is only one seeder in this simplistic protocol and the number of leechers is known and does not change, there is no need for a third party, i.e., a tracker.

${ }^{4}$ This holds because the protocol treats the rounds integrally. Otherwise, we could split a sufficiently big file into $M^{K}$ pieces for some $K$ and achieve a slightly reduced, optimal download time of $M+\frac{M^{2}}{M^{K}}$ using an analog algorithm. 


\subsection{Review of Game-Theoretic Definitions}

A game $\Gamma=\left(\left\{A_{i}\right\}_{i=1}^{n},\left\{u_{i}\right\}_{i=1}^{n}\right)$ consists of players $1, \ldots, n$ where each of them has a set $A_{i}$ of possible actions to play and individual utility functions $u_{i}$. All actions are played simultaneously; afterwards, every player $i$ receives a payoff that is determined by applying its utility function $u_{i}$ to the vector of actions played in the game.

Recent work has extended the traditional notion of a game to the requirements of cryptographic settings with their probabilistically generated actions and computationally-bounded running times. The resulting definition - called computational game [12] - allows each player $i$ to decide on a probabilistic polynomial-time (in the security parameter) interactive Turing machine $M_{i}$ (short PPITM). The machine $M_{i}$ is called the strategy for player $i$. The output of $M_{i}$ in the joint execution of these interactive Turing machines denotes the action of player $i$.

Definition 1 (Computational Game). Let $k$ be the security parameter and $\Gamma=\left(\left\{A_{i}\right\}_{i=1}^{n},\left\{u_{i}\right\}_{i=1}^{n}\right)$ a game. Then $\Gamma$ is a computational game if the played action $A_{i}$ of each participant $i$ is computed by a PPITM $M_{i}$ and if the utility $u_{i}$ of each player $i$ is polynomial-time computable.

Because of the probabilistic strategies, the utility functions $u_{i}$ now correspond to the expected payoffs. Rationally behaving players aim to maximize these payoffs. In particular, if a player knew which strategies the remaining players intend to choose, he would hence pick the strategy that induces the most beneficial outcome of the game. As this simultaneously holds for every player, we are looking for a so-called Nash equilibrium, i.e., a strategy vector where each player has no incentive to deviate from, provided that the remaining strategies do not change. Similar to the notion of a game, we consider a computational variant of a Nash equilibrium.

Definition 2 (Computational Nash Equilibrium). Let $\Gamma=\left(\left\{A_{i}\right\}_{i=1}^{n}\right.$, $\left.\left\{u_{i}\right\}_{i=1}^{n}\right)$ be a computational game and $k$ the security parameter. A strategy vector consisting of PPITMs $M=\left(M_{1}, \ldots, M_{n}\right)$ is a computational Nash equilibrium if for all $i$ and any PPITM $M_{i}^{\prime}$ there exists a negligible function $\epsilon$ such that $u_{i}\left(k, M_{i}^{\prime}, \boldsymbol{M}_{-i}\right)-u_{i}(k, \boldsymbol{M}) \leq \epsilon(k)$ holds.

Here $u_{i}\left(k, M_{i}^{\prime}, \boldsymbol{M}_{-i}\right)$ denotes the function $u_{i}$ applied to the setting where every player $j \neq i$ sticks to its designated strategy $M_{j}$ and only player $i$ deviates by choosing the strategy $M_{i}^{\prime}$.

We finally define the outcome of a computational game as the transcript of all players' inputs and the actions each has taken. In contrast to strategy vectors, an outcome thus constitutes a finished game where every player can determine its payoff directly. A utility function is thus naturally defined on the outcome of a computational game: When applied to a strategy vector (with its probabilistic choices), it describes the vector's expected payoff; when applied to an outcome of the game, it describes the exact payoff for this outcome. 


\subsection{A Game-Theoretic Model for File Sharing Protocols}

We now define the utility functions for seeders and leechers such that these functions characterize rational behavior in a file sharing protocol. We start by introducing common notation and some preliminaries.

Notation and Preliminaries. Following the BitTorrent convention, we call a player in the file sharing game a peer. The peers are divided into two groups: A seeder uploads to other peers a file $f$ that it owns, whereas a leecher downloads $f$. To mediate the communication among peers, we require a trusted party called the tracker. The tracker holds a signing key pair $(p k, s k)$, and we assume that its IP address and public key $p k$ are known to all peers.

The file $f$ consists of pieces $f_{1}, \ldots, f_{N}$, each of length $B$ bytes. The participants in the file sharing protocol hold the values $h_{1}=h\left(f_{1}\right), \ldots, h_{N}=h\left(f_{N}\right)$, where $h$ is a publicly known hash function. When deployed in practice, this publicly known information is distributed via a metainfo file. The tracker is only responsible for coordinating peers that are exchanging the same file. In order to stay close to a realistic setting, we allow different peers to have different upload and download capacities. Every seeder $S_{i}$ has its individual upload speed $u p_{i}^{s}(t, o)$ that depends on the time $t$ and the outcome $o$. Note that a seeder does not download anything except for metadata; hence we do not consider the download speed of seeders in this paper. Similarly, every leecher $L_{i}$ has individual upload and download speeds up $p_{i}^{l}(t, o)$ and $d o w n_{i}^{l}(t, o)$. We denote by $T_{i, \text { fin }}(o)$ the total time that leecher $L_{i}$ spends downloading the file. To increase readability, we omit the outcome in all formulas whenever it is clear from the context. We also introduce the sets $L=\left\{i \mid L_{i}\right.$ is a leecher $\}$ and $S=\left\{i \mid S_{i}\right.$ is a seeder $\}$.

Rationally-behaving Seeders. A seeder uploads parts of the file to other peers without requesting reciprocation. Intuitively, a seeder is interested in using as efficiently as possible its upload time and upload speed. Thus for any fixed upload speed and time that the seeder spends within the system, the average download time for all leechers should be as small as possible. We express this preference by the following seeder's utility function.

Definition 3 (Seeder's Utility Function). We say that $u_{i}^{s}$ is a utility function for a seeder $S_{i}$ if for any two outcomes o, of of the game with the same fixed upload speed up $p_{i}^{s}$ and fixed time $T_{i}^{s}$ spent by $S_{i}$ in the system, it holds that $u_{i}(o) \geq u_{i}\left(o^{\prime}\right)$ if and only if $\frac{1}{|L|} \sum_{i \in L} T_{i, \mathrm{fin}}(o) \leq \frac{1}{|L|} \sum_{i \in L} T_{i, \mathrm{fin}}\left(o^{\prime}\right)$.

If $S_{i}$ is the first seeder in the system, we thus require that $S_{i}$ uploads the whole file at least once. Otherwise, it is not rational to share the file in the first place.

Rationally-behaving Leechers. Leechers aim at downloading the shared file as fast as possible; moreover, they also try to use as little upload capacity as possible. The relative weight of these two (typically contradictory) goals is given by a parameter $\alpha_{i}$ in the system measuring time units per capacity units, e.g., seconds per kilobytes. 
Definition 4 (Leecher's Utility Function). Let $\alpha_{i} \geq 0$ be a fixed value. We say that $u_{i}^{l}$ is a utility function for leecher $L_{i}$ if the following condition holds: For two outcomes o, $o^{\prime}, L_{i}$ prefers outcome o to $o^{\prime}$ if and only if

$$
\alpha_{i} \cdot \int_{0}^{T_{i, \text { fin }}(o)} u p_{i}^{l}(t, o) d t+T_{i, \text { fin }}(o) \leq \alpha_{i} \cdot \int_{0}^{T_{i, \text { fin }}\left(o^{\prime}\right)} u p_{i}^{l}\left(t, o^{\prime}\right) d t+T_{i, \text { fin }}\left(o^{\prime}\right) .
$$

The value $\alpha_{i}$ corresponds to $L_{i}$ 's individual valuation for upload speed and time; e.g., if $\alpha_{i}=0.5$, the leecher values time twice as much as the uploaded data.

In particular, this definition implies that a rationally-behaving leecher prioritizes completing the download over everything else: If the leecher does not download the file in outcome $o$, then $T_{i, \text { fin }}(o)$ equals infinity. If it downloads the file in some outcome $o^{\prime}$, then $T_{i \text {,fin }}\left(o^{\prime}\right)$ is finite and thus increases its payoff.

\section{The RatFish Protocol}

We now present the RatFish protocol. We start with the description of the tracker and proceed with the seeders and leechers, respectively.

\subsection{The Protocol of the Tracker}

Similar to BitTorrent, our tracker manages all valid IP addresses in the system and introduces new leechers to a set of neighbors. However, we assign the tracker additional tasks: First, our tracker is responsible for awarding each newcomer with seeding capacity equivalent to $\gamma$ file pieces (for a tunable parameter $\gamma$ ) 5 Second, our tracker keeps track of which file pieces each peer owns at any given moment. This bookkeeping will be crucial for incentivizing peers to follow the RatFish protocol, for computing the deserved rewards and for answering queries about the leechers' availabilities. Third, the tracker imposes a forced wait for every leecher upon connecting, thereby preventing leechers from gaining advantages by frequently disconnecting and rejoining the protocol. Finally, if a leecher wishes to disconnect, the tracker provides a certificate on the most recent set of pieces the leecher has to offer. This allows leechers to later reconnect to RatFish and use their partially downloaded data, i.e., in order to cope with network disruptions. In the following, we describe the individual subprotocols of the tracker in detail. A rigorous description is given in Fig. 1.

The Connect Protocol. The tracker assigns every new leecher $L_{i}$ a random subset of size $H$ of all leechers that are currently in the system. This random subset corresponds to $L_{i}$ 's local neighborhood. The tracker sends this neighborhood information to $L_{i}$ after $T$ seconds. Once the forced wait is over, the leecher may start downloading $\gamma$ free pieces from seeders. The rationale behind

\footnotetext{
${ }^{5}$ In practice, $\gamma$ is a small constant number just big enough for the new leecher to participate in the system. As long as the seeders can provide $\gamma$ pieces to each newly joining leecher, this value does not influence the existence of the Nash equilibrium.
} 


\section{TrackerConnect (peer)}

If peer is a seeder $S_{i}$, receive the seeder's upload speed $u p_{i}^{s}$ and store it. Else, do:

- If a message "PIECES $\left(a_{1}, \ldots, a_{N}, i d_{\mathrm{r}}, s i g_{t^{\prime}}\right)$ " is received from $L_{i}$, verify that $s_{i} g_{t^{\prime}}$ is a valid signature on $\left(a_{1}, \ldots, a_{N}, i d_{\mathrm{r}}\right)$ for verification key $p k$ and that $p=\left(a_{1}, \ldots, a_{N}, i d_{\mathrm{r}}\right)$ was previously stored. In this case, remove $p$ from storage and set $A_{i}^{m}:=a_{m}$ for all $m \in\{1, \ldots, N\}$. Otherwise select a random $i d_{\mathrm{r}}$.

- As soon as the current time $T_{c}$ is larger than $T+T_{p}$, where $T_{p}$ is the connecting time of the leecher, i.e., after the forced wait of $T$ seconds, send $L_{i}$ a random subset of size $H$ of current leechers' IP addresses, corresponding to $L_{i}$ 's neighborhood. Moreover, compute $\mathrm{S}_{s k}\left(i, T_{p}\right)$, yielding a signature $s i g_{t}$. Send $\operatorname{TIME}\left(T_{p}, i d_{\mathrm{r}}, \operatorname{sig}_{t}\right)$ to $L_{i}$.

\section{CheckExchange}

Do the following steps unless one of their checks fail; abort in this case:

- Upon receiving a message $\operatorname{HAS}(j, y)$ from a leecher $L_{i}$, send back yes if $A_{j}^{y}=1$, and no otherwise.

- Upon receiving a message $\operatorname{EXCHANGED~}(j, x, y)$ from a leecher $L_{i}$, indicating that the pieces $x$ and $y$ have been exchanged with $L_{j}$, check $A_{i}^{x}=1$ and $A_{j}^{y}=$ 1. Send the message ACKNOWLEDGE $(i, x, y)$ to $L_{j}$.

- Upon subsequently receiving the message $\operatorname{OK}(i, x, y)$ from $L_{j}$, set $X_{i}:=X_{i}+1$ and $A_{i}^{y}:=1$, and send the message $\operatorname{OK}(j, x, y)$ to $L_{i}$.

RewardLeechers (called every $T$ seconds, i.e., at the start of a new round)

- Award $\gamma$ pieces to every leecher that joined in the previous round. Let prev be the number of these leechers.

- Compute for every leecher $L_{i}$ its deserved percentage of seeders' upload speed:

$$
r_{i}:=\min \left\{\frac{X_{i}}{\sum_{k \in L} X_{k}}, \frac{1}{2}\right\}
$$

- Let $\beta_{i}:=r_{i} \cdot\left(\frac{\sum_{k \in S} u p_{k}^{s} \cdot T}{B}-\gamma \cdot p r e v\right)$. For every $i$, determine a set of seeders that can jointly offer $\beta_{i}$ new pieces to $L_{i}$ such that the individual upload capacity of the seeders is respected, see Sect. 4.1. Send to every seeder the corresponding set of leechers and the number of pieces that these leechers should download from them.

- Set $A_{j}^{y}:=1$ if a seeder already informed the tracker that $y$ was downloaded by $S_{j}$. Set $X_{i}:=0$ for all $i$.

\section{PeerLeave(i)}

- Compute $s i g_{p}:=\mathrm{S}_{s k}\left(A_{i}^{1}, \ldots, A_{i}^{N}, i d_{\mathrm{r}}\right)$. Store $\left(A_{i}^{1}, \ldots, A_{i}^{N}, i d_{\mathrm{r}}\right)$.

- Send the message $\operatorname{LEAVE}\left(\operatorname{sig}_{p}\right)$ to $L_{i}$ and disconnect from $L_{i}$.

Fig. 1. The protocol of the tracker. The variable $A_{i}$ used in these protocols represents an array that stores which file pieces $L_{i}$ has already downloaded. The algorithm ensures that $A_{i}^{m}=1$ if leecher $L_{i}$ has the $m$-th piece of $f$, and $A_{i}^{m}=0$ otherwise. 


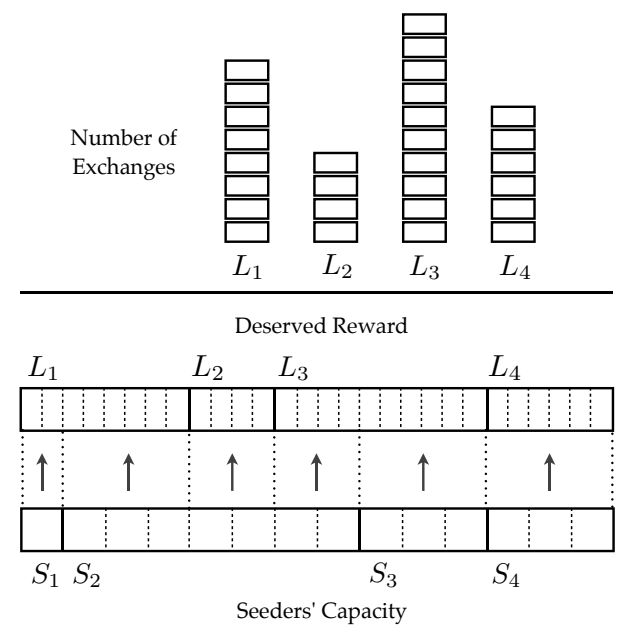

Fig. 2. Schematic distribution of the rewards

this forced wait is that granting newly joined leechers free pieces creates incentives for whitewashing, i.e., frequent disconnecting and rejoining. Intuitively, the forced wait is a simple defense against such a behavior. From a rational perspective, if a leecher joins the system only once, the induced small delay will not be hurtful; however, whitewashing by frequently rejoining will cause an accumulated delay that will result in a smaller payoff. The forced wait is achieved by having the tracker sign the leecher's connecting time and IP address. Such signed timestamps are exchanged between neighboors and are used to determine whether leechers are allowed to start uploading to eachother. Neighbors use the timestamps to determine whether they are allowed to start uploading to each other. Thus as long as a user's IP address does not change, it can idle and become active again without being penalized by a forced wait, since the user's old signature on its IP address and time is still valid.

The RatFish tracker has a mechanism for proving availability of rejoining leechers: it chooses a random rejoin ID $i d_{\mathrm{r}}$ and signs it together with the departing leecher's availability. The leecher uses $i d_{\mathrm{r}}$ to prove its availability upon rejoining the system. The rejoining $i d_{\mathrm{r}}$ is then deleted from the tracker's memory preventing leechers from reconnecting twice using the same reconnect $i d_{\mathrm{r}}$.

The Reward Protocol. The reward system constitutes the crucial part of RatFish. The underlying idea is to reward only leechers who are exchanging. We only allow one exception to this rule: The leechers that connected to the tracker in the previous round are also entitled to a reward of $\gamma$ pieces in the current round. Thus the seeders do not automatically upload to their neighborhood as in BitTorrent; rather they are told by the tracker whom to upload to.

To determine whether an exchange between $L_{i}$ and $L_{j}$ has happened, the tracker asks both $L_{i}$ and $L_{j}$ to acknowledge the exchange. If the acknowledgements succeed, the tracker internally increases the variables $X_{i}$ and $X_{j}$, which 


\section{Seeding $_{j}$}

Upon connecting, the seeder sends its upload speed $u p_{j}^{s}$ to the tracker. In each round, do:

- Receive from the tracker a set $M$ of leechers and the corresponding number of pieces $\omega_{i}$ that every leecher $L_{i} \in M$ should receive.

- Inform every leecher $L_{i} \in M$ how many pieces $\omega_{i}$ they are allowed to download.

- When a leecher $L_{i} \in M$ request at most $\omega_{i}$ pieces by $L_{i}$ (potentially incrementally in this round, i.e., it may ask for a few pieces first), send these pieces to $L_{i}$ and send a message to the tracker that these pieces have been uploaded to $L_{i}$. Requests by leechers $L_{j} \notin M$ are ignored.

Fig. 3. The protocol of the seeder $S_{j}$.

corresponds to the number of file piece exchanges of $L_{i}$ and $L_{j}$, respectively. The tracker moreover stores which pieces of the file the leechers now additionally know. Details on the participation of the tracker in the exchange protocol are given in Sect.4.3, where the exchange of pieces between leechers is explained.

Every round, i.e., after $T$ seconds, the actual rewards are given out. The tracker distributes the seeders' upstream in proportion to the number of exchanges every leecher made. Hence, the more exchanges a leecher completed, the larger the reward it obtains from the tracker, and hence the more download capacity it receives from the seeders. A graphical illustration of the reward protocol is given in Fig. 2 .

\subsection{The Protocol of the Seeder}

Upon connecting, the seeder informs the tracker about the upload speed it is going to offer. The tracker adds the seeder's speed to the amount of free available upload capacity. As the tracker performs all the computations for determining the rewards, the seeder simply proceeds by uploading the number of file pieces to the leechers as instructed by the tracker. To keep the tracker's information about individual leechers up-to-date, the seeder informs the tracker whenever it uploads a piece to a leecher. A rigorous description is given in Fig. 3 .

\subsection{The Protocol of the Leecher}

From a rational perspective, the leecher protocol is the most difficult to get right: while the tracker is honest and seeders partially altruistic, a leecher tries to bypass the incentives for uploading wherever reasonable.

Leechers can use the signed messages from the tracker to verify each other's join times. Also, when two leechers exchange data, the tracker participates in this exchange: Before two leechers start an exchange, they verify with the tracker that the other party holds the desired piece. If this check succeeds, two encryptions of 
the pieces are exchanged. Before they also send the key to each other to decrypt these messages, both leechers acknowledge the exchange to each other so that they get a higher reward.

The Connect Protocol. When a leecher connects to the tracker for the first time it requests a local neighborhood. If the leecher rejoins, it additionally proves to the tracker that it already owns some pieces of the file by sending the signature received from the tracker at its last disconnect. When connecting to a

LeecherConnect $_{i}$ (party)

If party is the tracker, distinguish two cases:

1. If $L_{i}$ rejoins the protocol, send $\operatorname{PIECES}\left(a_{1}, \ldots, a_{N}, i d_{\mathrm{r}}, s i g_{p}\right)$ to the tracker where $a_{m}=1$ if $L_{i}$ owns the $m$-th piece of the file, $i d_{\mathrm{r}}$ is the rejoin ID and $\operatorname{sig}_{p}$ is the signature received when disconnecting from system last time. If $L_{i}$ is a new leecher, it sends $\operatorname{PIECES}(0, \ldots, 0, \epsilon, \epsilon)$.

2. Receive $\operatorname{TIME}\left(T_{p}, i d_{\mathrm{r}}, s_{i} g_{t}\right)$ from the tracker - indicating the signed connecting time and ID, as well as a set of neighbors' IP addresses. Connect to them.

If party is a leecher $L_{j}$, do (abort if a check fails):

- Send the message MYTIME $\left(T_{p}\right.$, sigt $)$ to $L_{j}$.

- Receive the message $\operatorname{MYTIME}\left(T_{p}^{\prime}\right.$, siggt $)$ from $L_{j}$. Verify that $s i g_{t}^{\prime}$ is a valid signature on $\left(j, T_{p}^{\prime}\right)$ for $p k$ and that $T_{c}>T_{p}^{\prime}+T$ holds.

- Send AVAILABILITY $\left(a_{1}, \ldots, a_{N}\right)$ to $L_{j}$ where $a_{m}=1$ if $L_{i}$ owns $f_{m}$.

- Receive the message AVAILABILITY $\left(a_{1}^{\prime}, \ldots, a_{N}^{\prime}\right)$ from $L_{j}$.

\section{LeecherAwarded}

Whenever $L_{i}$ is informed by a seeder $S_{j}$ that it can download $\omega_{i}$ pieces, request up to $\omega_{i}$ pieces from $S_{j}$ (potentially incrementally in this round, i.e., $L_{i}$ may ask for a few pieces first), and download these pieces.

\section{Exchange $_{i}\left(f_{x}, j, y\right)$}

If any of the following checks fails, blacklist $L_{j}$ and abort.

- Send the message $\operatorname{HAS}(j, y)$ to the tracker and wait for a positive answer.

- Choose a random key $k_{j, x}$ and compute $c_{j, x} \leftarrow \mathrm{E}\left(k_{j, x}, f_{x}\right)$.

- Send $c_{j, x}$ to $L_{j}$ and wait for $c_{y}$ from $L_{j}$.

- Perform the following two steps in parallel and proceed once both steps are completed:

- Send $\operatorname{EXCHANGED}(j, x, y)$ to the tracker and wait for $\mathrm{OK}(j, x, y)$ as response

- If receiving $\operatorname{ACKNOWLEDGE}(j, y, x)$ from the tracker, reply with $\mathrm{OK}(j, y, x)$.

- Send the key $k_{j, x}$ to $L_{j}$.

- Upon receiving $k_{y}$ from $L_{j}$, retrieve $f_{y}^{\prime} \leftarrow \mathrm{D}\left(k_{y}, c_{y}\right)$ and verify $h_{y}=h\left(f_{y}^{\prime}\right)$.

- Broadcast to the local neighborhood that you now own the piece $y$.

Fig. 4. The protocol of the leecher $L_{i}$ 
seeder, the leecher requests pieces until its seeder's reward is depleted. Upon contacting another leecher, it waits until both forced waits are over. Afterwards, both parties exchange information such that they know which pieces they can request from each other. To keep track of the availability in its neighborhood, the leecher observes the messages that leechers broadcast to their local neighborhood, indicating which pieces of the file they have just downloaded.

The Piece Exchange. The piece exchange protocol run between two leechers uses encryptions to ensure that no leecher can get a piece without completing the exchange phase. From a practical perspective, it is important to note that the key sizes are small compared to a file piece size. Thus the communication and storage overhead induced by the keys and cryptographic operations is kept manageable. Leechers additionally query the tracker to ensure that the corresponding party owns a file piece they need. Moreover, leechers want their exchanges to be counted and rewarded. Thus, after the encryptions are exchanged, each leecher prompts the tracker to ask the other leecher for an acknowledgement. Intuitively, there is no incentive to deviate in this step as they still lack the key from the other party. Once the acknowledgement step is successfully completed, both leechers exchange the keys. If one leecher deviates from this procedure, it is blacklisted by the other leecher. We stress that blacklisting is not required for the security proof; it solely constitutes a common technique in this setting to deal with malicious parties. A rigorous description is given in Fig. 4. Fair exchange protocols have been used in prior work to incentivize peers to fairly exchange information [27. In contrast to [27, however, RatFish needs to neither periodically renew cryptographic keys nor implement a non-repudiable complaint mechanism to allow parties to prove possible misbehaviors; instead it relies on short acknowledgment messages for each recipient and on collecting these messages to monitor the file completion for the participants. A schematic overview of the core part of the piece exchange protocol is provided in Fig. 5.

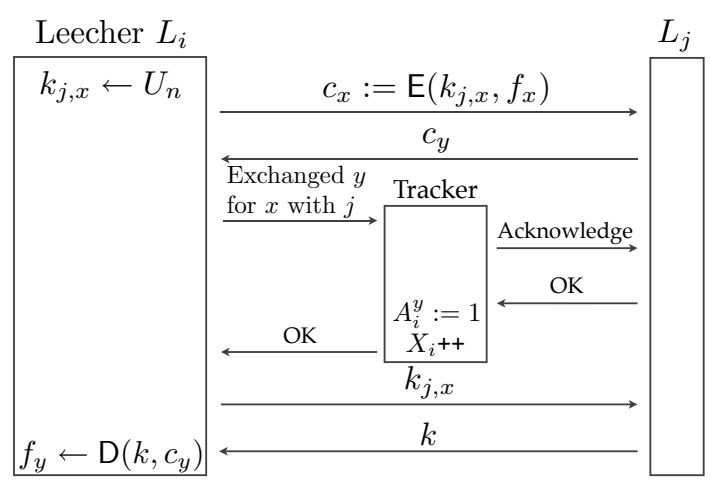

Fig. 5. The core part of the piece exchange protocol between two leechers 


\section{Equilibrium Proof}

In this section we prove that RatFish yields a computational Nash equilibrium; i.e., no leecher or seeder has an incentive to deviate from the protocol.

\subsection{Underlying Assumptions}

Recall that RatFish proceeds in rounds of $T$ seconds. For simplicity, we assume that peers can join / leave only at the beginning / end of a round and that it is impossible to forge identities on the IP layer (e.g., by using appropriate authentication mechanisms). We assume that at least one seeder is present and that the overall seeding capacity does not exceed twice the overall upload capacity of the leechers; this bound on the seeding capacity prevents the leechers from free riding, which is easy given enough seeding power. We moreover assume that each leecher's dedicated upload speed $u p_{i}^{l}$ is fully exhausted by other peers. These assumptions seem reasonable as the average seeders/leechers ratio is often close to 1:1 4, and optimized centralized coordinators are capable of distributing upload capacities among different swarms [20]. Additionally, we assume keys do not contribute to the uploaded amount, since in practice, the size of the short keys is dominated by the size of the encrypted file piece. Moreover, we assume that each peer is only able to maintain one identity in the system. This in particular excludes Sybil attacks, in which multiple distinct identities are created by the same peer to subvert the reputation system of a $\mathrm{P} 2 \mathrm{P}$ network. This assumption does not come as a surprise, since the Nash equilibrium conceptually does not defend against coalitions, rendering Sybil attacks possible in most works on rationally secure protocols. (See Section 7 for an outlook on how to tackle this problem.) Regarding the cryptographic primitives, we assume that the signature scheme used by the tracker is secure against existential forgery under chosen-message attack and that the encryption scheme is semantically secure under chosen-plaintext attack.

\subsection{Proving the Nash Equilibrium}

We finally show that RatFish constitutes a Nash equilibrium. Due to space constraints, we concentrate on illustrating how the proof is conducted and on highlighting the novel proof technique that was used. The technical parts of the proof are given in the full version.

We first show that a leecher deviating from the protocol cannot increase its utility by more than at most a negligible value, provided that no other party deviates. To show this, we determine two sets of possible cheating actions for leechers, which we call independent actions and correlated actions. Intuitively, the independent cheating actions can be individually replaced by honest actions

${ }^{6}$ This assumption can be easily enforced by letting the tracker force joining peers to wait until the next round. 
without decreasing the utility, independent of the remaining parts of the deviating strategy. Correlated cheating actions are sensitive to the details of the deviating strategy: we can only replace a correlated cheating action by a corresponding honest action without decreasing the utility if all deviating actions that jointly influence the leecher's utility are simultaneously replaced in one round. We show that the only correlated cheating action is to not acknowledge an exchange.

Our proof technique starts with an arbitrary deviating strategy $M_{i}^{\prime}$ and provides a proof in two steps: In the first step, we replace all independent cheating actions step-by-step; here, a step within a strategy denotes the computation performed within the strategy between two consecutive outputs. Slightly more formally, let $M_{i}$ be the honest strategy for leecher $L_{i}, M_{i}^{\prime}$ a deviating strategy, and $\left\{H_{a c k, j}^{*}\right\}_{j}$ the set of all strategies that in every step are honest or do not acknowledge an exchange. Then our technique yields a so-called semi-honest strategy $M_{i}^{*} \in\left\{H_{a c k, j}^{*}\right\}_{j}$ that for every input and random tape outputs in every step the same action as $M_{i}^{\prime}$ whenever possible, and plays honest otherwise. We then show that the semi-honest strategy cannot yield a worse payoff than $M_{i}^{\prime}$. The proof is based on the novel concept of hybrid concatenation of strategies.

Lemma 1 (No Independent Cheating Actions of Leechers). Let $\gamma$ be the number of uploaded pieces a newly joined leecher is awarded. Let $M_{i}^{\prime}$ be a deviating strategy of $L_{i}$ and let $M_{i}^{*}$ be the semi-rational strategy as defined above. Then for $\alpha_{i} \in\left[0, \frac{T}{3 \cdot \gamma \cdot B}\right]$, we have $u_{i}\left(k, M_{i}^{\prime}, \boldsymbol{M}_{-i}\right)-u_{i}\left(k, M_{i}^{*}, \boldsymbol{M}_{-i}\right) \leq \epsilon(k)$ for some negligible function $\epsilon$.

Thus far, we have transformed a deviating strategy $M_{i}^{\prime}$ into a semi-rational strategy $M_{i}^{*}$ that uses only correlated cheating actions and does not decrease the payoff. In the second step, we replace all correlated cheating actions roundby-round until we reach the honest strategy $M_{i}$. We use a hybrid argument based on the hybrid concatenation of strategies to show that the honest strategy outperforms the semi-rational strategy for leechers.

Lemma 2 (No Correlated Cheating Actions of Leechers). Let $M_{i}$ be the honest strategy for $L_{i}$, i.e., following the RatFish protocol and let $M_{i}^{*}$ be the semirational strategy as defined above. Then $u_{i}\left(k, M_{i}^{*}, M_{-i}\right)-u_{i}\left(k, M_{i}, M_{-i}\right) \leq \epsilon(k)$ holds for some negligible function $\epsilon$.

Showing that seeders have no incentive to deviate from the protocol is considerably easier than the corresponding statement for leechers, since seeders are considered partially altruistic. We show that as long as all leechers follow the protocol, a seeder cannot run a deviating strategy to improve its payoff.

Lemma 3 (No Seeder Deviation). There is no deviating strategy for any seeder that increases its payoff if all other parties follow the RatFish protocol.

We finally combine the results that neither leechers nor seeders have an incentive to deviate (the tracker is honest by assumption) to establish our main result.

Theorem 1 (Computational Nash Equilibrium). The RatFish protocol constitutes a computational Nash equilibrium if $\alpha_{i} \in\left[0, \frac{T}{3 \cdot \gamma \cdot B}\right]$ holds for all $i \in L$. 


\section{Implementation and Performance Evaluation}

In this section, we describe the implementation of RatFish and experimentally evaluate its performance. We focus on the implementation and performance evaluation of the tracker, since the tracker took on several additional responsibilities and is now involved in every exchange. In contrast to the tracker, seeders and leechers are largely unchanged when compared to BitTorrent: the exchange of encrypted pieces constitutes a small computational overhead, but leaves the network complexity that usually constitutes the performance bottleneck of P2P protocols essentially unchanged.

\subsection{Implementation}

The RatFish tracker was implemented using about 5000 lines of code in Java, thus ensuring compatibility with common operating systems. The implementation is designed to work with both UDP and TCP.

The messages sent in the protocol start with the protocol version number and message ID (which determines the length of the message), followed by the torrent ID, and additional information that depends on the type of message.

Afterwards, a task is created that processes the received message. This task is given to the threadpool executor - the main part of the RatFish tracker that also ensures parallelization. The threadpool sustains eight parallel threads and assigns new tasks to the next free thread. For instance, when the tracker receives a notification that a leecher joined the protocol, the task extracts the leecher's IP from this message and triggers the forced wait. After $T$ seconds it replies with a digital signature for the leecher using an RSA-based signature scheme that signs SHA-1 hashes.

\subsection{Experimental Setup}

For the evaluation, we ran the RatFish tracker on a server with a 2-cores Intel Xeon CPU, 2GB of RAM, a 100MBit Internet connection and an Ubuntu SMP operating system with kernel version 2.6.28-18. We simulated a swarm with up to 50,000 peers, divided into neighborhoods of size 4 . The simulated leechers send the same messages as a real leecher would, thus yielding an accurate workload measure for the tracker. Every leecher was configured to complete one exchange per second, and we chose the size of a piece to be $256 \mathrm{kB}$ according to BitTorrent standards. Hence every leecher has a virtual upload speed of $256 \mathrm{kB} / \mathrm{s}$. The size of the shared file is $50 \mathrm{MB}$, and the seeders upload one forth of the file per second in a round-robin fashion to their neighborhoods. The simulated clients are running on a separate machine. This allows us to measure network throughput. In our simulation, we need to pretend to the tracker that clients connect from different IPs. We thus used UDP in our experiments. Deploying RatFish in reality would presumably be based on TCP, which would slightly increase the network complexity. 


\subsection{Performance Evaluations}

Fig. 6] depicts the results for our experiments. The main observation, shown in the left part of Fig. 6, is that even though we engage the tracker in every exchange in the swarm, the protocol scales well (a resource usage of $65 \%$ for 50,000 leechers). One can also observe that the computation complexity becomes a limiting factor, but we expect this to change for more cores given our highly parallelized implementation. Memory was not a bottleneck in any experiment.

The right part of Fig. 6 considers the case where many leechers join at once, but no exchanges are happening. This study is important since the tracker's most expensive task is to sign the join time of leechers. In our experiments, the tracker was able to serve about 400 new leechers per second. Since the server has $T$ seconds for signing in practical deployments, the signature computation would be scheduled in free CPU time and hence not delay ongoing exchanges. We also observed that the two measurements depicted in Fig. 6 on CPU usage are additive, e.g., a system with 30,000 leechers and 200 joining leechers per second uses approximately $90 \%$ of the CPU.

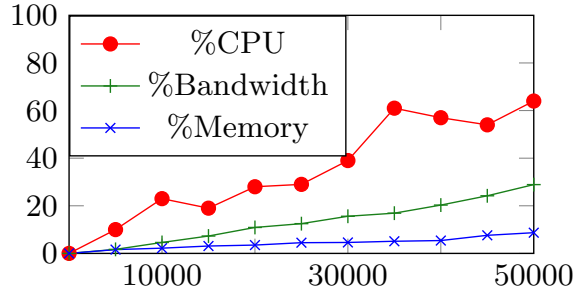

Joining leechers per swarm

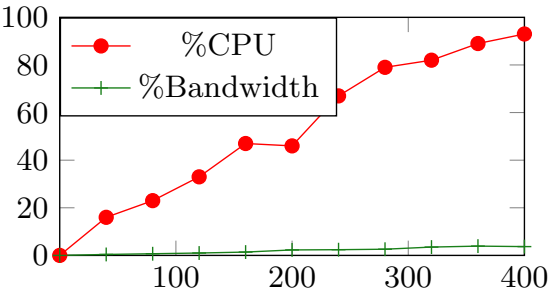

Joining leechers per second

Fig. 6. Left: Resource usage for a static number of leechers engaging in exchanges. Right: Resource usage for dynamically joining leechers.

\section{Conclusions and Future Work}

We have proposed a file sharing protocol called RatFish that we have proven secure against deviations of rational users. We first characterized rational behaviors of leechers and seeders in file sharing protocols. Subsequently, we formally showed that no rational party has an incentive to deviate from RatFish; i.e., RatFish constitutes a Nash equilibrium. While the tracker in RatFish is assigned additional tasks compared to existing file sharing protocols such as BitTorrent, the communication overhead of a RatFish client compared to a BitTorrent client is minimal. We have demonstrated by means of a prototype implementation that RatFish is practical and efficient.

A central question for future work on rational file sharing - and for rational cryptography in general - is whether the Nash equilibrium is a strong enough notion for real-world applications and threat models. Robustness against user 
coalitions would be more desirable. (See the discussion in [7] and [1].) RatFish already provides suitable hooks for potential mitigation techniques against coalitions, e.g., by ensuring that players entering small coalitions can only increase their utilities by a negligible amount; hence entering a coalition would be irrational in the first place. Moreover, RatFish currently considers file sharing for independent swarms only, i.e., seeders in one swarm cannot be leechers in another swarm. Extending RatFish to cope with such more a general setting requires to generalize the seeders' utility functions and to adapt the relevant parts of RatFish in order to maintain the Nash equilibrium property.

\section{References}

1. Abraham, I., Dolev, D., Gonen, R., Halpern, J.: Distributed computing meets game theory: robust mechanisms for rational secret sharing and multiparty computation. In: 25th Annual ACM Symposium on Principles of Distributed Computing (PODC 2006), pp. 53-62 (2006)

2. Aiyer, A.S., Alvisi, L., Clement, A., Dahlin, M., Martin, J.P., Porth, C.: BAR fault tolerance for cooperative services. Operating Systems Review (OSR) 39(5), 45-58 (2005)

3. Bharambe, A.R., Herley, C., Padmanabhan, V.N.: Analyzing and improving a BitTorrent network's performance mechanisms. In: The 25th IEEE Conference on Computer Communications, INFOCOM 2006 (2005)

4. Bieber, J., Kenney, M., Torre, N., Cox, L.P.: An empirical study of seeders in BitTorrent. Tech. rep., Duke University (2006)

5. Cohen, B.: Incentives build robustness in BitTorrent. Tech. rep., bittorrent.org (2003)

6. Dodis, Y., Halevi, S., Rabin, T.: A cryptographic solution to a game theoretic problem. In: Bellare, M. (ed.) CRYPTO 2000. LNCS, vol. 1880, pp. 112-130. Springer, Heidelberg (2000)

7. Feigenbaum, J., Shenker, S.: Distributed algorithmic mechanism design: recent results and future directions. In: 6th International Workshop on Discrete Algorithms and Methods for Mobile Computing and Communications (DIAL-M 2002), pp. 1-13 (2002)

8. Fuchsbauer, G., Katz, J., Naccache, D.: Efficient rational secret sharing in standard communication networks. In: Micciancio, D. (ed.) TCC 2010. LNCS, vol. 5978, pp. 419-436. Springer, Heidelberg (2010)

9. Gordon, D., Katz, J.: Rational secret sharing, revisited. In: De Prisco, R., Yung, M. (eds.) SCN 2006. LNCS, vol. 4116, pp. 229-241. Springer, Heidelberg (2006)

10. Halpern, J., Teague, V.: Rational secret sharing and multiparty computation: extended abstract. In: STOC 2004, pp. 623-632 (2004)

11. Izal, M., Uroy-Keller, G., Biersack, E., Felber, P.A., Hamra, A.A., Garces-Erice, L.: Dissecting BitTorrent: Five months in torrent's lifetime. In: Barakat, C., Pratt, I. (eds.) PAM 2004. LNCS, vol. 3015, pp. 1-11. Springer, Heidelberg (2004)

12. Katz, J.: Bridging game theory and cryptography: Recent results and future directions. In: Canetti, R. (ed.) TCC 2008. LNCS, vol. 4948, pp. 251-272. Springer, Heidelberg (2008)

13. Keidar, I., Melamed, R., Orda, A.: Equicast: Scalable multicast with selfish users. Computer Networks 53(13), 2373-2386 (2009) 
14. Kol, G., Naor, M.: Cryptography and game theory: Designing protocols for exchanging information. In: Canetti, R. (ed.) TCC 2008. LNCS, vol. 4948, pp. 320 339. Springer, Heidelberg (2008)

15. Levin, D., LaCurts, K., Spring, N., Bhattacharjee, B.: BitTorrent is an auction: analyzing and improving BitTorrent's incentives. Computer Communications Review (CCR) 38(4), 243-254 (2008)

16. Li, H.C., Clement, A., Marchetti, M., Kapritsos, M., Robison, L., Alvisi, L., Dahlin, M.: FlightPath: Obedience vs. choice in cooperative services. In: USENIX OSDI 2008, pp. 355-368 (2008)

17. Li, H.C., Clement, A., Wong, E.L., Napper, J., Roy, I., Alvisi, L., Dahlin, M.: BAR gossip. In: USENIX OSDI 2006, pp. 191-204 (2006)

18. Liogkas, N., Nelson, R., Kohler, E., Zhang, L.: Exploiting BitTorrent for fun (not for profit) (2006), http://iptps06.cs.ucsb.edu/

19. Locher, T., Moor, P., Schmid, S., Wattenhofer, R.: Free riding in BitTorrent is cheap. In: 5th Workshop on Hot Topics in Networks (HotNets-V), pp. 85-90 (2006)

20. Peterson, R.S., Sirer, E.G.: Antfarm: efficient content distribution with managed swarms. In: USENIX NSDI 2009, pp. 107-122 (2009)

21. Piatek, M., Isdal, T., Anderson, T., Krishnamurthy, A., Venkataramani, A.: Do incentives build robustness in BitTorrent? In: USENIX NSDI 2007, pp. 1-14 (2007)

22. Piatek, M., Isdal, T., Krishnamurthy, A., Anderson, T.: One hop reputations for peer to peer file sharing workloads. In: USENIX NSDI 2008, pp. 1-14 (2008)

23. Pouwelse, J.A., Garbacki, P., Epema, D., Sips, H.J.: The BitTorrent p2p file-sharing system: Measurements and analysis. In: Castro, M., van Renesse, R. (eds.) IPTPS 2005. LNCS, vol. 3640, pp. 205-216. Springer, Heidelberg (2005)

24. Qiu, D., Srikant, R.: Modeling and performance analysis of BitTorrent-like peerto-peer networks. In: SIGCOMM 2004, pp. 367-378 (2004)

25. Rob, D.L., Sherwood, R., Bhattacharjee, B.: Fair file swarming with FOX (2006), http://iptps06.cs.ucsb.edu/

26. Shneidman, J., Parkes, D., Massoulie, L.: Faithfulness in internet algorithms. In: Workshop on Practice and Theory of Incentives and Game Theory in Networked Systems (PINS 2004), pp. 220-227 (2004)

27. Sirivianos, M., Yang, X., Jarecki, S.: Robust and efficient incentives for cooperative content distribution. Transactions On Networking (TON) 17(6), 1766-1779 (2009)

28. Thommes, R., Coates, M.: BitTorrent fairness: Analysis and improvements. In: 4th IEEE Workshop on the Internet, Telecommunications and Signal Processing, WITSP 2005 (2005) 